(C) 1982. The Genetical Society of Great Britain

\title{
PUPAL COLOUR DIMORPHISM IN SWALLOWTAIL BUTTERFLIES AS A THRESHOLD TRAIT: SELECTION IN EURYTIDES MARCELLUS (CRAMER)
}

\author{
WADE N. HAZEL* and DAVID A. WEST \\ Department of Biology, Virginia Polytechnic Institute and State University, Blacksburg, \\ VA 24061, U.S.A.
}

Received 10.xi.81

\section{SUMMARY}

The pupal colour dimorphism of Eurytides marcellus is nearly lost when the species is forced to pupate on a clear plastic substrate. Selection for extreme brown and green pupal colour under these circumstances was successful in a single generation in 3 out of 5 and 7 out of 10 lines respectively, but selection for an intermediate colour gave no response in 3 out of 3 lines. The results support a threshold model of pupal colour dimorphism in this species, with "sensitivity" to substrate characteristics being the underlying continuous variable. The consequently strong environmental component of variation in pupal colour in natural populations thus serves to maintain the genetic variation, since natural selection will be only weakly stabilizing.

\section{INTRODUCTION}

THE pupae of many species of swallowtail butterfies are dimorphic, being either green or brown. However, unlike many polymorphisms in the adult stages of the Lepidoptera, pupal colour is to a large degree environmentally controlled (Merrifield and Poulton, 1899; Sheppard, 1958).

The significance of the environmental influence on pupal colour is thought to lie in the crypsis it provides the sedentary and exposed pupae (Sheppard, 1958; Wiklund, 1975). Thus, prepupal larvae respond to various environmental cues while on their pupation sites by producing the appropriate pupal colour: green on or amid green foliage and brown on or amid brown foliage, twigs, etc. (Smith, 1978; Hazel and West, 1979).

Two hypotheses have been proposed to explain the genetic basis of pupal colour dimorphism. Clarke and Sheppard (1972) suggested that individuals differ in their genetic tendencies to produce cryptic pupae. Alternatively, Hazel (1977) suggested that individuals differ in their genetic tendencies to produce either green or brown pupae. Thus, an individual with a high tendency to produce a brown pupa would have a low tendency to produce a green pupa. The hypotheses are conceptually similar in that both envision continuous genetic variation underlying the two pupal colours, but they differ in their views of the nature of the genetic differences among individuals. As a result, they give different predictions of the response of the trait to selection. If individuals differ in their genetic tendencies to produce cryptic pupae, then brown pupae on green surfaces should be genetically similar to green pupae on brown surfaces, and selection of either class of individuals should increase the frequency of such U.S.A

* Present address: Department of Zoology, DePauw University, Greencastle, IN 46135 , 
non-cryptic pupae. In nature, selection would be strongly directional, resulting in a uniformly high tendency of individuals to produce cryptic pupae. By contrast, if individuls differ simply in their tendencies to produce green or brown pupae, then brown pupae on green surfaces and green pupae on brown surfaces should be genetically different. In nature, selection would be weakly stabilizing, instead of directional. Individuals with a high tendency to produce brown pupae would sometimes pupate on brown substrates and individuals with a high tendency to produce green pupae would sometimes pupate on green foliage. That is, despite their extreme genotypes, they would often produce adaptive (cryptic) phenotypes. As a result, genetic variation underlying the pupal colours would be maintained.

Both hypotheses have been tested experimentally. Clarke and Sheppard (1972) allowed two greenhouse populations of Papilio polytes to pupate on natural surfaces and then artificially selected cryptic pupae in one population and non-cryptic pupae in the other. After approximately five generations of selection they were still unable to detect a response. Hazel (1977) artificially selected in $P$. polyxenes for the tendencies of individuals to produce green or brown pupae on a given population substrate. Although the sample sizes were small, there was a response to selection in one generation in the brown line and in two generations in the green line.

In this paper we report the results of selection for pupal colour in the swallowtail Eurytides marcellus. These results provide additional evidence that the genetic basis of pupal colour dimorphism lies in differences among individuals in their tendencies to produce green and brown pupae, and that these differences are maintained in nature by weak stabilizing selection.

\section{MATERIALS AND METHODS}

Pairings were made to form a base population with adults that were one generation removed from the wild. Larvae of both the base and the selected populations were reared on a $15 \mathrm{~L}: 9 \mathrm{D}$ photoperiod, at a constant temperature of $24^{\circ} \mathrm{C}$, in round plastic boxes and were fed fresh pawpaw leaves (Asimina triloba) daily. Other details of rearing are given by Hazel (1977).

Within 30 minutes of entering the prepupal stage, as indicated by gut evacuation, larvae of the base population were placed in clear plastic rearing boxes on a white surface, illuminated with constant fluorescent light supplemented by daylight from an adjacent window. Prepupal larvae of the selected generation were placed in clear plastic rearing boxes, either uncovered or covered on the outside with green construction paper and suspended above a white surface so that light entered from below. Larvae from all broods were placed in these two types of pupation environments.

The pupae of $E$. marcellus are cryptic in nature, with green pupae being formed on the undersides of green leaves and brown pupae on the undersides of brown leaves. In our field studies we have yet to observe any non-cryptic or intermediately coloured pupae (West and Hazel, in prep.). In the laboratory, distinct dimorphism continues to be the rule for pupations on nearly all pupation sites, whether artificial (coloured plastic and paper) or natural (logs and twigs). However, when pupation occurs on clear plastic the dimorphism is nearly lost and pupae vary almost continuously in colour from green to brown. To form criteria for artificial selection, we classified 
pupae into five phenotypic categories, based mainly on the degree of brownness (table 1). To keep our observations consistent we used the same phenotypic classification for pupae formed in plastic rearing dishes covered with green construction paper, although these pupae were less variable in colour.

TABLE 1

Classification of pupal colour phenotypes of Eurytides marcellus pupating on clear plastic in constant light

\begin{tabular}{ll}
\multicolumn{1}{c}{ Phenotype } & \multicolumn{1}{c}{ Description } \\
Green-1 & green; cuticle opaque \\
Green-2 & green; cuticle translucent \\
Green-intermediate & $\begin{array}{l}\text { pale green; brown in thicker areas of cuticle; } \\
\text { cuticle opaque or translucent } \\
\text { tan; lacking brown spots on dorsal abdominal } \\
\text { segments; cuticle opaque or translucent } \\
\text { Brown-intermediate } \\
\text { abdo brown, with brown spots on dorsal } \\
\text { abdominal segments; cuticle opaque }\end{array}$
\end{tabular}

Three selected lines were formed by hand pairing 18 pairs of butterflies. Ten pairings between adults eclosing from green-1 pupae comprised the green-1 line. Three pairings between adults eclosing from green intermediate pupae formed the green intermediate line. The brown line consisted of five pairings between adults eclosing from brown pupae. No green-2 or brown intermediate pairings were made because all of these pupae entered diapause. Inbreeding was strictly avoided and selection was only practiced for one generation.

\section{Results}

Table 2 shows the results of each selected pairing, giving the midparent and $F_{1}$ frequencies of the selected pupal colour phenotypes formed on clear plastic, and statistical comparisons of these frequencies. For example, the broods from which the parents of brood 18 were drawn produced a total of $17 \cdot 7$ per cent green-1 pupae, while selection resulted in a significant increase in the frequency of green-1 pupae in brood 18 to 34.1 per cent. Comparisons of the frequencies of selected pupal colours in the midparent and $F_{1}$ generation were tested by the $\mathrm{G}$-statistic. Of the ten broods comprising the green-1 line, seven showed a significant increase, two no change, and one a significant decrease in the frequency of green-1 pupae in the $F_{1}$ generation. Of five broods selected for brown, three showed a significant increase, one no change and one a significant decrease, in the frequency of brown. The midparent/offspring regression for pupation on clear plastic is 0.54 but lacks significance $(P>0 \cdot 10)$. A sign test of the numbers of significant increases and decreases gives $P<0 \cdot 02$. None of the three broods selected for green-intermediate showed a significant change in that phenotype.

Table 3 shows the frequencies of brown pupae produced on clear and green-backed plastic by each $F_{1}$ brood. The correlation of per cent brown pupae per brood on the two substrates in the selected generation is significant (arc-sine transformation, $r=0 \cdot 75, n=18, P<0 \cdot 01$ ). 
TABLE 2

Results of selection for pupal colour in E. marcellus, showing midparent and $F_{1}$ frequencies of the selected pupal colour, family size and $G$-statistics for $2 \times 2$ tables

\begin{tabular}{|c|c|c|c|c|c|c|c|}
\hline $\begin{array}{l}\text { Selection } \\
\text { Criterion }\end{array}$ & Brood & Midparent $\%(\mathbf{N})$ & $\mathrm{F}_{1} \%$ & $(\mathrm{~N})$ & Response & G & $\mathbf{P}$ \\
\hline Green-1 & $\begin{array}{r}18 \\
1 \\
2 \\
5 \\
11 \\
4 \\
4 \\
14 \\
15 \\
6 \\
17\end{array}$ & $\begin{array}{rr}17 \cdot 7 & (79) \\
18.3 & (120) \\
18.3 & (120) \\
23.2 & (95) \\
23.2 & (95) \\
34.5 & (87) \\
43.4 & (53) \\
48.2 & (166) \\
57.6 & (59) \\
59.6 & (89)\end{array}$ & $\begin{array}{l}34 \cdot 1 \\
46 \cdot 3 \\
35 \cdot 6 \\
42 \cdot 7 \\
20 \cdot 7 \\
63 \cdot 5 \\
73 \cdot 2 \\
81 \cdot 6 \\
23 \cdot 1 \\
48 \cdot 3\end{array}$ & $\begin{array}{l}(41) \\
(67) \\
(87) \\
(75) \\
(29) \\
(52) \\
(41) \\
(38) \\
(65) \\
(29)\end{array}$ & $\begin{array}{l}+ \\
+ \\
+ \\
+ \\
0 \\
+ \\
+ \\
+ \\
- \\
0\end{array}$ & $\begin{array}{r}3 \cdot 84 \\
14 \cdot 9 \\
7 \cdot 8 \\
7 \cdot 3 \\
0 \cdot 1 \\
11 \cdot 0 \\
8 \cdot 4 \\
14 \cdot 8 \\
15 \cdot 6 \\
1 \cdot 1\end{array}$ & $\begin{aligned} & 0.05 \\
< & 0.001 \\
< & 0.01 \\
< & 0.01 \\
> & 0.70 \\
< & 0.001 \\
< & 0.01 \\
< & 0.001 \\
< & 0.001 * \\
> & 0.20\end{aligned}$ \\
\hline Brown & $\begin{array}{r}8 \\
20 \\
21 \\
22 \\
13\end{array}$ & $\begin{array}{l}43 \cdot 6(181) \\
43.6(181) \\
46 \cdot 0(150) \\
46 \cdot 0(150) \\
47 \cdot 2(159)\end{array}$ & $\begin{array}{l}67 \cdot 3 \\
29 \cdot 0 \\
33 \cdot 3 \\
77 \cdot 8 \\
91 \cdot 4\end{array}$ & $\begin{array}{l}(55) \\
(62) \\
(36) \\
(36) \\
(23)\end{array}$ & $\begin{array}{l}+ \\
- \\
0 \\
+ \\
+\end{array}$ & $\begin{array}{r}9 \cdot 5 \\
4 \cdot 2 \\
1 \cdot 9 \\
12 \cdot 2 \\
17 \cdot 8\end{array}$ & $\begin{array}{l}<0.01 \\
<0.05^{*} \\
>0.10 \\
<0.001 \\
<0.001\end{array}$ \\
\hline $\begin{array}{l}\text { Green- } \\
\text { intermediate }\end{array}$ & $\begin{array}{r}3 \\
7 \\
10\end{array}$ & $\begin{array}{ll}19 \cdot 5 & (87) \\
19 \cdot 5 & (87) \\
19.5 & (87)\end{array}$ & $\begin{array}{r}21 \cdot 5 \\
9 \cdot 4 \\
28 \cdot 1\end{array}$ & $\begin{array}{l}(65) \\
(32) \\
(32)\end{array}$ & $\begin{array}{l}0 \\
0 \\
0\end{array}$ & $\begin{array}{l}0.1 \\
1.8 \\
0.9\end{array}$ & $\begin{array}{l}>0.70 \\
>0.10 \\
>0.30\end{array}$ \\
\hline
\end{tabular}

* Wrong response.

TABLE 3

Frequencies of brown pupae in selected broods on two pupation substrates

\begin{tabular}{|c|c|c|c|c|}
\hline Brood & \multicolumn{2}{|c|}{ Clear plastic $(\mathrm{N})$} & \multicolumn{2}{|c|}{ Green-backed plastic (N) } \\
\hline 18 & $41 \cdot 5$ & (41) & 51.7 & (29) \\
\hline 1 & $37 \cdot 3$ & (67) & $66 \cdot 7$ & (48) \\
\hline 2 & $23 \cdot 0$ & (87) & $32 \cdot 9$ & (76) \\
\hline 5 & $22 \cdot 7$ & (75) & $43 \cdot 8$ & (28) \\
\hline 11 & $31 \cdot 0$ & (29) & $91 \cdot 3$ & (23) \\
\hline 4 & $19 \cdot 2$ & (52) & $46 \cdot 7$ & (30) \\
\hline 14 & $9 \cdot 8$ & (41) & $41 \cdot 4$ & (29) \\
\hline 15 & $15 \cdot 8$ & (38) & $57 \cdot 7$ & (26) \\
\hline 6 & 55.4 & (36) & $78 \cdot 8$ & (52) \\
\hline 17 & $31 \cdot 0$ & (29) & $90 \cdot 9$ & (22) \\
\hline 8 & $67 \cdot 3$ & (55) & $65 \cdot 2$ & $(42)$ \\
\hline 20 & $29 \cdot 0$ & (62) & $63 \cdot 0$ & (54) \\
\hline 21 & $33 \cdot 3$ & $(36)$ & $65 \cdot 6$ & (32) \\
\hline 22 & $77 \cdot 8$ & (36) & $87 \cdot 1$ & (27) \\
\hline 13 & $91 \cdot 4$ & (23) & 100 & (14) \\
\hline 3 & $13 \cdot 8$ & (65) & 28.8 & $(52)$ \\
\hline 7 & $25 \cdot 0$ & (32) & $52 \cdot 0$ & (25) \\
\hline 10 & $37 \cdot 5$ & (32) & 73.9 & (23) \\
\hline
\end{tabular}

\section{Discussion}

The results support a threshold model for pupal colour dimorphism in Eurytides marcellus, with individuals differing genetically in the levels of their thresholds for producing brown pupae ("sensitivities", Hazel 1977). According to the model, individual threshold level (sensitivity) is continuously variable, but different environments (pupation sites) set the critical 
level at different points along the distribution of sensitivities, giving different proportions of green and brown pupae. Families may also differ in their mean sensitivities (Hazel, 1977). Fig. 1 illustrates the two ways in which the proportions of green and brown pupae can be changed. Different pupation environments can alter the position of the critical threshold (Figs. $1 \mathrm{a}$ and $\mathrm{b}$ ), or selection can change the position of the mean relative to the critical threshold for a particular pupation environment (Figs. 1c and d). Our results show both situations.
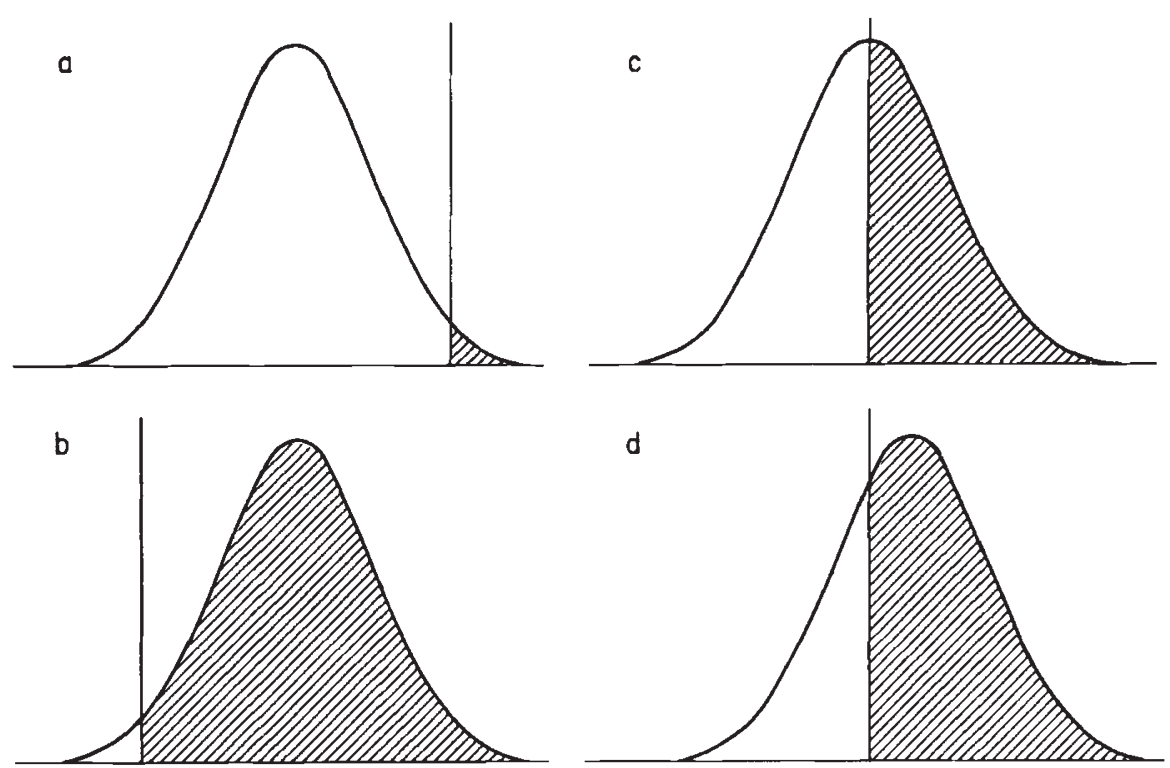

INCREASING SENSITIVITY $\longrightarrow$

FIG. 1.-Demonstration of how the frequencies of brown (cross-hatched) and green pupae (clear) can be altered environmentally, by imposition of the population threshold at different points relative to the mean sensitivity of the population, (a) for a green pupation site, (b) for a brown pupation site; or genetically, by altering the mean sensitivity of the population via selection, (c) parental generation, (d) selected generation on same pupation site as parental generation.

If a trait controlled by one set of genes is measured in two environments, then in the absence of a genotype-environment interaction the two measurements will be correlated (Falconer 1960, Chapter 19). For pupal colour, this predicts a correlation between the frequencies of brown pupae produced by members of a brood on two kinds of pupation sites. The significant correlation of per cent pupae per brood on clear plastic and on plastic backed by green paper shows that although these two pupation environments set the critical threshold at different points in the distribution, the same set of genes underlies the expression of pupal colour in both environments.

The results of positive assortative mating of brown and of green-1 phenotypes illustrate how selection can alter the mean of genetic sensitivities in relation to a fixed critical threshold. The lack of response 
to selection in the green-intermediate line is consistent with the view that these individuals are of intermediate sensitivities and, assuming complete additivity, are heterozygous at many of the loci affecting the trait. Thus selection of these individuals does not change the mean sensitivity.

We have considered pupal colour in $E$. marcellus to be the discontinuous expression of continuous genetic variation because in most pupation environments the pupal colour in this species is discontinuous. There is some variation within greens and browns, but intermediates are very rare (Hazel, 1980). The clear plastic pupation sites used in the present experiments evidently failed to set a crisp critical threshold level. E. marcellus is unlike other swallowtails in our experience in that its pupal colour is not affected by variation in the texture of the pupation site, only by its colour (Hazel, 1980). Without a colour cue, the plastic may have given the larvae ambiguous signals, and those individuals having intermediate sensitivities responded with intermediate pupal colours. In other swallowtails clear plastic gives a sufficient textural cue to allow the expression of the brown/green dichotomy.

There is an important difference between artificial and natural selection for pupal colour. Artificial selection in our experiments was for differences in genetic sensitivity as revealed by differences in pupal colour. In nature, selection is for pupal colour as it relates to the pupation site (degree of crypsis). With regard to the genetic basis of pupal colour this means that the larvae most likely to produce cryptic pupae are those of intermediate genetic sensitivity. Because those individuals are capable of the greatest phenotypic flexibility, they will produce cryptic pupae over the widest range of pupation sites. The larvae least likely to produce cryptic pupae will be those lying at the extremes of the distribution of genetic sensitivities. These individuals, being the least flexible phenotypically, will produce cryptic pupae over only a narrow range of pupation sites: insensitive larvae only on green pupation sites; sensitive larvae only on brown pupation sites. As a result, selection in nature will be stabilizing at the genotypic level while appearing to be directional (favouring crypsis) or even disruptive (favouring both green and brown pupae) at the phenotypic level. Clarke and Sheppard's (1972) selection for cryptic pupae was therefore stabilizing at the genotypic level, and its failure to change the incidence of crypsis is understandable.

Since Clarke and Sheppard's (1972) experiment, while failing to affect pupal colour, did alter the pupation site preference of larvae, it is worth asking what effect pupation site preference may have on the way that natural selection acts on the genetic basis of pupal colour. According to our model, in a population where green and brown pupation sites are equally available to, and used by, prepupal larvae, genotypes of intermediate sensitivity are most fit, as they will produce cryptic pupae on both green and brown pupation sites.

If brown sites become more common, or are preferred by prepupal larvae, natural selection will favour the more sensitive extreme genotypes over the less sensitive because they will produce cryptic pupae more often. Although genotypes of intermediate sensitivity are still the most likely to produce cryptic pupae, the difference in incidence of crypsis between extreme sensitive and insensitive genotypes will result in a change in the mean sensitivity of the population in the direction of increased sensitivity. 
This will be reflected in an increased tendency to produce brown pupae on all pupation sites. Thus, selection will be directional until the mean sensitivity of the population has reached the point where the extreme sensitive and insensitive genotypes are equally likely to produce cryptic pupae. When this new mean has been reached, selection will once again be stabilizing. Therefore, our model predicts that the mean sensitivity of a population, as reflected by its tendency to produce brown pupae over a range of pupation sites, will be correlated with its relative use of pupation sites in nature where brown is the cryptic pupal colour. This prediction is borne out in Battus philenor, a species of swallowtail which strongly prefers brown pupation sites (West and Hazel, 1979, 1982) and which produces higher frequencies of brown pupae over a wide range of pupation sites than do other species of sympatric swallowtails (Hazel and West 1979). In California, Sims and Shapiro (unpublished results) find that $B$. philenor pupates more often on sites where green is the cryptic colour, and they show that the average sensitivity of prepupae to pupation site characteristics is lower than in $B$. philenor from Virginia.

It is of interest to note that in a situation where selection favours the use of a variety of pupation sites, it will also serve to maintain, by stabilizing selection, the genetic variation underlying pupal colour dimorphism. While we have some idea of the kinds of selective forces influencing differences in pupation site preferences between species (West and Hazel, 1982), we know little of how selection operates within a species to maintain variation in pupation site preference.

\section{REFERENCES}

CLARKE, C. A., AND SHEPPARD, P. M. 1972. Genetic and environmental factors influencing pupal colour in the swallowtail butterflies Battus philenor and Papilio polytes. J. Entomol., (A) 46, 123-136.

FALCONER, D. S. 1960. Introduction to quantitative genetics. Edinburgh and London, Oliver and Boyd.

HAZEL. W. N. 1977. The genetic basis of pupal colour dimorphism and its maintenance by natural selection in Papilio polyxenes (Papilionidae: Lepidoptera). Heredity, 38, 227-236.

HAZEL, W. N., AND WEST. D. A. 1979. Environmental control of pupal colour in swallowtail butterflies (Lepidoptera: Papilioninae): Battus philenor (L.) and Papilio polyxenes Fabr. Ecological Entomology, 4, 393-400.

HAZEL, W. N. 1980. The evolution and ecological genetics of pupal color dimorphism in swallowtail butterfies (Lepidoptera: Papilioninae). Ph.D. Thesis, Virginia Polytechnic Institute and State University, Blacksburg.

MERRIFIELD. F.. AND POULTON, E. B. 1899. The colour-relation between the pupae of Papilio machaon, Pieris napi and many other species, and the surroundings of the larvae, etc. Transactions of the Entomology Society of London, 1899, 369-433.

SHEPPARD, P. M. 1958 Natural Selection and Heredity. London, Hutchinson.

SMITH, A. G. 1978. Environmental factors influencing pupal colour determination in Lepidoptera. I. Experiments with Papilio polytes, Papilio demoleus and Papilio polyxenes. Proc. R. Soc. Lond., B., 200, 295-329.

WEST, D. A.. AND HAZEL. W. N. 1979. Natural pupation sites of swallowtail butterflies (Lepidoptera: Papilioninae): Papilio polyxenes Fabr., P. glaucus L. and Battus philenor (L.). Ecological Entomology, 4, 387-392.

WEST. D. A., AND HAZEL. W. N. 1982. An experimental test of natural selection for pupation site in swallowtail butterflies. Evolution, 36, 152-159.

WIKLUND, C. 1975. Pupal colour polymorphism and the survival in the field of cryptic versus non-cryptic pupae in Papilio machaon L. Trans. R. Ent. Soc. Lond., 127, 73-84. 